\title{
Quantifying the effects of combination trastuzumab and radiation therapy in human epidermal growth factor receptor 2 positive breast cancer
}

Meghan J Bloom ( $\sim$ meghanjbloom@gmail.com )

University of Texas at Austin

Patrick N Song

University of Alabama at Birmingham

John Virostko

University of Texas at Austin

Thomas E Yankeelov

University of Texas at Austin

Anna G Sorace

University of Alabama at Birmingham

\section{Research}

Keywords: HER2+, Herceptin, radiosensitive, BT474

Posted Date: June 23rd, 2020

DOI: https://doi.org/10.21203/rs.3.rs-30208/v2

License: (c) (1) This work is licensed under a Creative Commons Attribution 4.0 International License. Read Full License 


\section{Abstract}

Background: Trastuzumab, a clinical antibody targeted to the human epidermal growth factor receptor 2 (HER2), has been shown to sensitize cells to radiation in vitro. Current studies lack longitudinal evaluation of cellular response and in vivo data is limited. The purpose of this study is to quantify the effects of combination trastuzumab and radiation therapy in vitro and in vivo over time to determine if there is a synergistic interaction.

Methods: EGFP expressing BT474, SKBR3 and MDA-MB-231 cell lines were treated with $0.1 \mathrm{ng} / \mathrm{ml}$ of trastuzumab, 5 or $10 \mathrm{~Gy}$ of radiation, or combination treatment, and imaged using fluorescence live cell microscopy for one week. The Bliss independence model was used to quantify the effects of combination treatment. HER2+ tumor bearing mice (female $\mathrm{NU} / \mathrm{J})(\mathrm{N}=34)$ were treated with saline, $10 \mathrm{mg} / \mathrm{kg}$ of trastuzumab, 5 or 10 Gy of radiation, or combination treatment. Tumor size was measured three times per week for four weeks via caliper measurements. Additional mice $(\mathrm{N}=13)$ were treated with $10 \mathrm{mg} / \mathrm{kg}$ of trastuzumab, $5 \mathrm{~Gy}$ of radiation, or combination treatment. Tumors were harvested at one week and evaluated with immunohistochemistry for inflammation (CD45), vascularity (CD31 and a-SMA), and hypoxia (pimonidazole).

Results: Altering the order of therapies did not significantly affect BT474 cell proliferation in vitro $(P>0.05)$. The interaction index calculations revealed additive effects of trastuzumab and radiation treatment in all three cell lines in vitro. In vivo results revealed significant differences in tumor response between mice treated with 5 and 10 Gy single agent radiation $(P<0.001)$; however, no difference was seen in the combination groups when trastuzumab was added to the radiation regimen $(P=0.56)$, indicating a lower dose of radiation could be used without decreasing therapeutic efficacy. Histology results revealed increases in inflammation $(C D 45+)$ in mice receiving trastuzumab $(P<0.05)$.

Conclusions: Longitudinal evaluation of cell proliferation in vitro showed additive effects of combination therapy. In vivo results show a potential to achieve the same efficacy of treatment with reduced radiation when also administering trastuzumab. Further evaluation of tumor microenvironmental alterations during treatment could identify mechanisms of increased therapeutic efficacy in this regimen.

\section{Background}

Human epidermal growth factor receptor 2 positive (HER2+) breast cancer is characterized by overexpression of the HER2/neu receptor and represents $15-20 \%$ of breast cancer cases [1]. Trastuzumab, a monoclonal antibody targeted to the HER2 receptor, is used clinically in both neoadjuvant and adjuvant standard-of-care treatment, and is often combined with radiation in the adjuvant setting to eliminate residual disease and prevent recurrence [2-4]. Preclinical studies have shown that overexpression of HER2 is a contributing factor to radiation resistance, and treatment with anti-HER2 therapy could potentially act as a radiosensitizer [5-9]. However, HER2+ patients still have a greater chance of 
recurrence than patients with HER2-status [10]. Thus, there is a need to investigate optimizing trastuzumab and radiation in a combination treatment regimen.

One mechanism by which HER2 overexpression promotes tumorigenesis is through inhibition of apoptosis by upregulation of the phosphatidylinositol 3'-kinase-protein kinase B-mammalian target of rapamycin (PI3K/AKT/mTOR) pathway. Trastuzumab blocks PI3K/AKT/mTOR pathway signaling, causing cell cycle arrest and inducing cancer cell death [11]. Studies from Guo et al. [7] and Liang et al. [5] found that when used in combination, trastuzumab sensitizes HER2+ breast cancer cells to radiation therapy in vitro, and that the upregulation of PI3K/AKT pathway may be specifically involved in radioresistance in HER2+ breast cancer. Additionally, breast tumors can become hypoxic due to the irregular and tortuous vasculature which increases radioresistance [12-14]. Previous results in HER2+ breast cancer have revealed that treatment with trastuzumab can increase tumor oxygenation in a preclinical mouse model through stabilization of tumor blood vessels [17]. Although there has been investigation into the potential ability of trastuzumab to radiosensitize breast cancer, data quantifying sensitization over time has been lacking, thereby reducing clinical relevance. Furthermore, the effects of the order of therapies has not been systematically investigated [18].

Common in vitro approaches for evaluating cell response to radiation therapy include end point analyses such as the 3-[4,5-dimethylthiazolyl-2]-2,5-diphenyltetrazolium bromide (MTT) assay or the clonogenic assay. Studies evaluating cell viability after treatment can vary largely depending on the chosen end time point [19]. Additionally, these methods lack the ability to longitudinally observe cell response which is critical in determining underlying changes in cell physiology during therapy [20].

This contribution has two goals: 1 ) investigate and quantify the effects of radiation and trastuzumab longitudinally in vitro, 2) and test the hypothesis that trastuzumab sensitizes HER2+ breast cancer to radiation therapy in an in vivo model of HER2+ breast cancer. This study uses the IncuCyte Live-Cell Analysis System (Essen Bioscience, Ann Arbor, MI) which delivers real-time data of cellular responses and has been shown to be accurate in analyzing changes in breast cancer cell proliferation through time [21]. Quantifying the synergy between trastuzumab and radiation over time has the potential to elucidate optimal combination regimens and decrease the amount of therapy needed to achieve tumor control or eradication.

\section{Methods}

\section{Cell Culture}

All cell lines were purchased from the American Type Culture Collection (ATCC, Manassas, VA). BT474 cells were maintained in improved minimal essential medium (IMEM, Invitrogen, Carlsbad, CA) supplemented with $10 \%$ FBS, $1 \%$ penicillin/streptomycin, and $20 \mu \mathrm{g} / \mathrm{mL}$ of insulin. SKBR3 cells were cultured in McCoy's 5A medium (ATCC, Manassas, VA) supplemented with 10\% FBS and $1 \%$ 
penicillin/streptomycin. MDA-MB-231 cells were maintained in Dulbecco's Modified Eagle's Medium (Thermo Fisher Scientific Inc., Waltham, MA) supplemented with $5 \% \mathrm{FBS}$ and $200 \mathrm{mg} / \mathrm{mL}$ of G418. All cells were grown at $37^{\circ} \mathrm{C}$ with $5 \% \mathrm{CO}_{2}$. Cells were cultured to $70-80 \%$ confluency and cell counts were determined with the Countess II FL automated cell counter (Thermo Fisher Scientific Inc., Waltham, MA).

\section{Western Blot Evaluation of HER2 Expression and Quantification}

BT474, SKBR3, and MDA-MB-231 breast cancer cells were washed with ice cold phosphate buffered saline and lysed for 10 minutes on ice with radioimmunoprecipitation assay buffer supplemented with protease inhibitor (Roche Applied Science, Indianapolis, IN). Lysates were centrifuged at $13,400 \mathrm{~g}$ for 20 minutes at $4^{\circ} \mathrm{C}$ and collected for bicinchoninic acid protein quantification assay via the Nanodrop 2000c spectrophotometer (Thermo Fisher Scientific, Waltham, MA). For each cell line, $20 \mu \mathrm{g}$ of protein was prepared in a solution of sodium dodecyl sulfate and $\beta$-mercaptoethanol and boiled for 5 minutes at $95^{\circ}$ C. Samples were run on a NuPAGE Bis-Tris gel and transferred to a polyvinylidene fluoride membrane. The membrane was blocked in $5 \%$ dry milk in tris-buffered saline (TBST), probed with horseradish peroxidase (HRP) conjugated mouse anti-human $\beta$-actin overnight at $4^{\circ} \mathrm{C}$ and developed with the Amersham ECL western blot detection system (GE healthcare, Buckinghamshire, UK) for one minute at room temperature. Membranes were developed in the absence of light and visualized with an SRX-101A Medical Film Processor (Konica Minolta Medical and Garphic, Inc., Shanghai, China). The membrane was incubated in stripping buffer for 15 minutes, washed with TBST and probed with Rabbit anti-human HER2/ErbB2 primary antibody (Cell Signaling Technology, Danvers, MA) for 2 hours at room temperature. The membrane was washed, incubated with HRP conjugated goat anti-rabbit IgG secondary antibody (Cell Signaling Technology, Danvers, MA) for 1 hour at room temperature. After a final wash, the membrane was redeveloped and visualized for HER2 expression. Quantification of bands was conducted with the Image Studio Lite program, version 5.0 (LI-COR Biosciences, Lincoln, NE, USA). HER2 expression was normalized to $\beta$-actin expression for quantification.

\section{Transfection of Breast Cancer Cell Lines}

BT474, SKBR3, and MDA-MB-231 cells were transfected to express enhanced green fluorescent protein (EGFP) through the Sleeping Beauty Transposon System. An EGFP plasmid was obtained and cloned into a Sleeping Beauty compatible vector, psDBbi-Neo (Addgene, Cambridge, MA, plasmid \#60525). Lipofectamine LTX (Thermo Fisher Scientific, Waltham, MA) was used to co-transfect the psDBbi-Neo vector and the pCMV (CAT) T7-SB100 (Addgene, Cambridge, MA, plasmid \#34879) Sleeping Beauty transposase. Following transfection, cells were cultured in their respective media supplemented with $200 \mathrm{mg} / \mathrm{mL}$ G418 for four weeks to select for positively transfected cells. Fluorescence activated cell sorting was used to separate cells, and the $25 \%$ highest intensity EGFP expressing cells were used in the 
experiments. The pCMV (CAT)T7-SB100 plasmid was a gift from Dr. Zsuzsanna Izsvak [22] and the pSBbi-Neo was a gift from Dr. Eric Kowarz [23].

\section{In Vitro Treatment Experiments}

\section{Experiment 1: Order of dosing}

BT474-GFP cells were plated in 96-well plates at 5,000 cells/well. 24 hours later (Day 0), plates were placed in the IncuCyte S3 Live-Cell Analysis System (Essen BioScience, Ann Arbor, MI) and whole-well imaged every six hours with phase contrast and green channels (excitation 440-480, emission 504-544) for one week. Single agent trastuzumab treatment groups were treated with trastuzumab $(0.1 \mathrm{ng} / \mathrm{ml})$ from 0 to 48 hours (group 1), 24-72 hours (group 2), or 48-96 hours (group 3). For single agent radiation treatment groups, cells were treated with radiation (10 Gy) at 24 hours with the CellRad Dedicated Benchtop Cell Irradiator (Faxitron, Tucson, AZ). For combination treatment groups, cells received trastuzumab $(0.1 \mathrm{ng} / \mathrm{ml})$ before, with, or after radiation treatment (10 Gy) as detailed in Fig. 1a. Each treatment group has 24 replicates per experiment. The experiment was performed three times.

Experiment 2: Quantifying radiation + trastuzumab combination effects

BT474-GFP, SKBR3-GFP, or MDA-MB-231-GFP cells were plated in 96-well plates at 5,000 cells/well. 24 hours later (Day 0$)$, cells were treated with either $0.1 \%$ saline, trastuzumab $(0.1 \mathrm{ng} / \mathrm{ml})$, or radiation $(5$ or $10 \mathrm{~Gy}$ ) for control and single agent treatment groups. Combination groups received both trastuzumab $(0.1 \mathrm{ng} / \mathrm{ml})$ and radiation (5 or $10 \mathrm{~Gy}$ ) on Day 0 (Fig. 1b). Plates were placed in the IncuCyte S3 Live-Cell Analysis System (Essen BioScience, Ann Arbor, MI) and whole-well imaged (4×, 2.82 um/pixel) every six hours with phase contrast and green channels (excitation 440-480, emission 504-544) for one week. Each treatment group has 30 replicates per experiment. The experiments were performed three times.

\section{Image Analysis}

Images were analyzed using the IncuCyte S3 Live-Cell Analysis System (Essen BioScience, Ann Arbor, MI) to segment discrete green fluorescent objects (cell counts). Background signal was estimated in $100 \mu \mathrm{m}$ segments and subtracted from the image. Edges of clumped cells were determined to be the dimmest point between two objects. Cell growth was normalized to initial seeding density ( 0 hours).

\section{Interaction Index Calculation}


The interaction index [24] based on Bliss independence was used to determine effects of combination radiation and trastuzumab treatment in vitro. To calculate the effects of radiation $(A)$, trastuzumab $(B)$, and combination treatment $(A B)$ compared to the control, let $(f)$ represent the difference in normalized cell growth between control $\left(N_{C}\right)$ and treated $\left(N_{i}\right)$ conditions:

$$
\text { (1) } f_{i}=\frac{N_{C}-N_{i}}{N_{C}}
$$

where $i=A, B, A B$. The interaction index (I) can then be calculated by:

$$
\text { (2) } \quad I=f_{A B}-f_{A}-f_{B}+f_{A} f_{B} \text {, }
$$

where the value of $I$ determines the effect of combination therapy with the following interpretation

$$
\text { (3) } I=\left\{\begin{array}{cl}
\text { Synergistic } & I>0 \\
\text { Additive } & I=0 \\
\text { Antagonistic } & I<0
\end{array}\right.
$$

\section{Animal Procedures}

Female nude athymic mice $(\mathrm{NU} / \mathrm{J})(\mathrm{N}=47)$ were purchased from The Jackson Laboratory (Bar Harbor, ME) at 3-4 weeks of age at an average weight of 12-15 g, and acclimated for one week in housing with a normal light cycle, sterile water and food, and microisolation cages. Mice were subcutaneously implanted with a $0.72 \mathrm{mg}, 60$-day release, $17 \beta$-estradiol pellet (Innovative Research of America, Sarasota, $\mathrm{FL}$ ) in the nape of the neck. One day later, $10^{7}$ BT474 cells were implanted subcutaneously in $100 \mu \mathrm{l}$ of serum-free IMEM media with 30\% growth factor-reduced Matrigel. Tumors were grown to approximately $250 \mathrm{~mm}^{3}$ (8-10 weeks) and entered into the study. Animals were randomly sorted into treatment groups: group 1) Control: $100 \mu \mathrm{l}$ saline on Days $0,3(\mathrm{~N}=6)$, group 2) Trastuzumab alone: $10 \mathrm{mg} / \mathrm{kg}$ on Days 0,3 $(\mathrm{N}=7)$, group 3) Radiation 5 Gy alone: Day $0(\mathrm{~N}=6)+100 \mu$ saline on Day 3, group 4) Radiation 10 Gy alone: Day $0(\mathrm{~N}=5)+100 \mu$ saline on Day 3, group 5) Radiation 5 Gy on Day $0+$ Trastuzumab $10 \mathrm{mg} / \mathrm{kg}$ 
on Days 0, $3(\mathrm{~N}=5)$, group 6) Radiation 10 Gy on Day $0+$ Trastuzumab $10 \mathrm{mg} / \mathrm{kg}$ on Days $0,3(\mathrm{~N}=5)$. Trastuzumab and saline were administered via intraperitoneal (IP) injection. Radiation treatments ( 4 Gy/min, 225 kV, $17.8 \mathrm{~mA}$ ) were given with the MultiRad 350 Irradiation System (Faxitron, Tucson, AZ). Tumor measurements were taken three times per week using calipers for four weeks at which point mice were euthanized. Mice in the immunohistochemistry cohort were divided into three treatment groups: 1$)$ Radiation 5 Gy on Day $0(\mathrm{~N}=4)+100 \mu \mathrm{l}$ saline on Day 3, 2) Trastuzumab $10 \mathrm{mg} / \mathrm{kg}$ on Days $0,3(\mathrm{~N}=4)$, 3) Radiation 5 Gy on Day $0+$ Trastuzumab $10 \mathrm{mg} / \mathrm{kg}$ on Days $0,3(\mathrm{~N}=5)$. Tumor size was measured with calipers by measuring the longest axis of the tumor and the axis perpendicular to the longest axis. The minimum of these two measurements was assumed to be the height of the tumor to calculate the volume. Tumor size was measured three times for one week at which point mice were euthanized and tumors were extracted. One hour prior to sacrifice, mice were intravenously injected with $60 \mathrm{mg} / \mathrm{kg}$ of pimonidazole (Hypoxyprobe, Inc., Burlington, MA) in $100 \mu$ of saline. Tumors were cut at the longest cross-section and half was fixed in $10 \%$ neutral buffered formalin (Fisher Scientific International Inc., Pittsburgh, PA) for 48 hours where it was then placed in $70 \%$ ethanol.

\section{Immunohistochemistry}

Formalin fixed tumor sections were embedded in paraffin and sliced into $4 \mu \mathrm{M}$ sections. Sections were stained for hematoxylin and eosin (H\&E), mouse anti-CD31, mouse anti-a-smooth muscle actin ( $\mathrm{a}-$ SMA, Abcam, Cambridge, UK), mouse anti-CD45 (Invitrogen, Carlsbad, CA) and anti-pimonidazole (Hypoxyprobe, Inc., Burlington, MA). Immuno-stained slides were scanned (20x, $0.495 \mu \mathrm{m} /$ pixel) with the Aperio ScanScope (Leica Microsystems, Wetzlar, Germany). Regions of necrosis were determined using manual segmentation. All other stains were quantitatively segmented based on color thresholds determined from positive and negative controls using in house MATLAB (MathWorks Inc., Natick, MA) routines. Images were converted to grayscale and registered to the corresponding tumor H\&E via an intensity-based registration algorithm. A viable tissue mask was defined as total tumor area minus necrotic area. Inflammation (CD45), hypoxia (pimonidazole), and apoptosis (caspase3) were defined as the percent of positive stain per viable tissue area. Microvessel density (CD31) and vascular smooth muscle coverage ( $\mathrm{a}-\mathrm{SMA}$ ) were calculated as the number of vessels per $\mathrm{mm}^{2}$ of tumor tissue. The vessel maturation index [40] was evaluated as the ratio of a-SMA coverage to microvessel density.

\section{Statistical Analysis}

Statistical analysis was conducted using MATLAB. A two-way analysis of variance (ANOVA), adjusting for multiple comparisons with Dunn's, was used to determine longitudinal differences in order of dosing experiments. All in vitro data is presented as mean $\pm 95 \%$ confidence interval with $\mathrm{P}<0.05$ indicating significance. Statistical differences between in vivo tumor size at each time point as well as differences in ex vivo immunohistochemistry samples were determined using a nonparametric Wilcoxon 
rank sum test. All in vivo and ex vivo data is presented as mean \pm standard error with $\mathrm{P}<0.05$ indicating significance. The Friedman's test adjusting for multiple comparisons with Dunn's test was used to assess longitudinal changes in tumor growth between treatment groups.

\section{Results}

\section{HER2 expression in BT474, SKBR3, and MDA-MB-231 cell lines}

Quantification of HER2 protein expression in BT474, SKBR3, and MDA-MB-231 cells confirmed reported HER2 status in each cell line (Supp. Fig. 1). Western blot results revealed visible bands of HER2 protein in BT474 and SKBR3 HER2+ cell lines at $185 \mathrm{kDa}$, and showed little to no expression in MDA-MB-231 cells, a HER2- cell line (Supp. Fig. 1a). $\beta$-actin was used as an internal control for normalization of HER2 expression. Ratios of HER2: $\beta$-actin in BT474 and SKBR3 cells were 1.41 and 1.46 , respectively, confirming HER2 overexpression. MDA-MB-231 cells had a HER2: $\beta$-actin ratio of 0.08 , confirming no HER2 overexpression (Supp. Fig. 1b).

\section{Order of radiation and trastuzumab therapy does not affect cell death response in vitro}

Cells were administered with trastuzumab alone as a single agent control from either 0-48 hours, 24-72 hours, or 48-96 hours. No significant differences were observed between these groups (Fig. 2). In combination regimens, cells were treated with trastuzumab $(0.1 \mathrm{ng} / \mathrm{ml}) 24$ hours before radiation (10 Gy) treatment, at the same time as radiation treatment, and 24 hours after radiation treatment. No significant differences were seen in cell growth over time when comparing trastuzumab treatment before radiation with treatment at the same time $(P=1.00)$ or treatment after radiation $(P=0.98)$. Additionally, treatment with trastuzumab at the same time as radiation did not alter cell response over time compared to trastuzumab treatment after radiation $(P=0.93)$ (Fig. 2). As order of dosing did not significantly affect cell response to therapy, subsequent in vitro experiments were conducted administering trastuzumab and radiation at the same time for convenience (Fig. 1b).

\section{Quantification of longitudinal cell growth after combination therapy reveals additive effects in vitro}

Fig. 3 displays BT474, SKBR3, and MDA-MB-231 cell proliferation after being treated with trastuzumab $(0.01 \mathrm{ng} / \mathrm{ml})$, radiation ( 5 or $10 \mathrm{~Gy}$ ), or combination treatment over one week. No significant differences were observed through time in MDA-MB-231 cell proliferation between the control and trastuzumab single agent treatment groups $(P=0.88$, panels e and $f$ of Fig. 3$)$, which is to be expected in a HER2- cell line not responsive to trastuzumab. There were also no significant differences in proliferation between the radiation single agent or combination treatment groups as expected $(P=0.84$, Fig. 3e and $P=0.80$, Fig. 3f). Fig. 4 displays the interaction index over time for each treatment group in Fig. 3. No group had an interaction index that fell significantly above or below 0 at any time point $(P>0.05)$, indicating additive treatment effects. 
Fig. 5a displays tumor volume changes in response to trastuzumab, radiation, and combination regimens over four weeks. Mice treated with $10 \mathrm{~Gy}$ radiation had a significantly greater decrease in tumor size than mice treated with 5 Gy radiation over time $(P<0.001)$ (Fig. $5 b)$. However, mice treated with trastuzumab + 5 Gy radiation revealed no statistical differences in tumor response over time compared to mice treated with trastuzumab +10 Gy radiation $(P=0.56)(F i g .5 c)$. Additionally, mice treated with trastuzumab +5 Gy radiation had significantly smaller tumors than mice treated with trastuzumab alone from Day 7 onwards $(P<0.05)$ with the exception of Day $9(P=0.11)$. Mice treated with trastuzumab +5 Gy radiation had significantly smaller tumors than mice treated with $5 \mathrm{~Gy}$ alone from Day 2 onwards $(P<0.05)$ with the exception of Day $25(P=0.25)$. Mice treated with trastuzumab +5 Gy radiation had significantly smaller tumors than mice treated with 10 Gy radiation on Days 2-17 $(P<0.05)$.

\section{Tumor immune infiltration was higher in mice that received trastuzumab treatment}

Fig. 6a displays representative images of CD45+ staining in mice treated with radiation (5 Gy) on Day 0, trastuzumab $(10 \mathrm{mg} / \mathrm{kg})$ on Days 0 and 3 , or combination radiation and trastuzumab. Mice treated with trastuzumab alone had significantly higher CD $45+$ staining $(2.63 \pm 0.73 \%)$ than mice treated with radiation alone $(0.77 \pm 0.13 \%)(P=0.03)$ on Day 7 . Mice treated with combination therapy also had significantly higher $C D 45+$ staining $(2.48 \pm 0.44 \%)$ than mice treated with radiation alone $(P=0.03)$. No significant differences were observed between mice treated with trastuzumab alone and combination treatment $(P=1.00)$ (Fig. 6b). No significant differences were observed between treatment groups $(P>$ 0.05) in percent pimonidazole (Supp. Fig. 2a) or vascular maturation index (Supp. Fig. 2b).

\section{Discussion}

Trastuzumab and radiation are commonly used as adjuvant therapies in the clinical treatment of HER2+ breast cancer. Radiation therapy can reduce recurrence rates in patients by eradicating remaining cancer cells after surgical removal of tumors; however, HER2+ patients have greater chances of recurrence than HER2- patients [10, 25, 26]. In vitro evidence using end point assays suggests trastuzumab can sensitize HER2+ breast cancer cells to radiation through inhibition of signaling pathways involved in DNA repair mechanisms [5, 27], although longitudinal data systematically evaluating the order of therapies and the associated cellular response are limited. The present study demonstrates that, in vitro, the order of trastuzumab and radiation treatment does not yield statistically significantly different cell responses, and their longitudinal treatment effects are additive. Furthermore, in vivo, tumors regress faster when treated with combination therapy than either single agent therapy and there is evidence that immune modulation may impact treatment response.

The interaction index calculations revealed additive effects from combination radiation and trastuzumab treatment in both HER2+ breast cancer cell lines evaluated. This contrasts with others who have evaluated cell response to combination radiation and trastuzumab using end point assays [5, 9]. For example, Liang et al. found a synergistic effect from trastuzumab and radiation treatment with BT474 and SKBR3 cells 24 hours after therapy using an ELISA to evaluate apoptosis (cytoplasmic histone- 
associated DNA fragments) [5]. In their study, cells were pre-incubated with trastuzumab for 16 hours before radiation treatment; however, our results did not indicate that pre-treatment with trastuzumab alters cell death to radiation. Rao et al. found a similar synergistic effect using SUM-149PT cells treated with a HER2 tyrosine-kinase inhibitor in combination with radiation therapy; though, their study used a longer pre-incubation time with trastuzumab ( 7 days) and evaluated cell growth two weeks after radiation treatment using a clonogenic assay [9]. Differences in results could arise from the alternate methods used to detect cellular response to treatment, different dosing strategies, and differences in the timing of evaluation after treatment. One important aspect of our study is that we have quantified longitudinal cell growth after variations of combination treatments, thereby providing a better understanding of response dynamics after combination treatment compared to traditional end point assays. This provides the opportunity to assess long-term effects of the order of sequencing of therapies and variations in dosing strategies.

In vivo tumor growth data shows significant differences in longitudinal tumor response between groups that were treated with $5 \mathrm{~Gy}$ and $10 \mathrm{~Gy}$ of radiation; however, no significant difference in longitudinal tumor response was observed between the $5 \mathrm{~Gy}$ and $10 \mathrm{~Gy}$ doses when trastuzumab was added to the treatment regimen. This finding suggests a smaller dose of radiation could be used in combination with trastuzumab without decreasing efficacy. Additionally, results show a faster rate of tumor regression in groups treated with combination therapy compared to either single therapy.

To evaluate if microenvironmental alterations could be impacting treatment response, immune infiltration, hypoxia, and the vascular maturation index were evaluated in tumors treated with $5 \mathrm{~Gy}$ radiation, trastuzumab, and $5 \mathrm{~Gy}$ radiation in combination with trastuzumab seven days after treatment. CD45 histology staining revealed an increase in immune infiltration in treatment groups receiving trastuzumab. Previously collected data revealed trastuzumab has the potential to reprogram the immunosuppressive components of the tumor microenvironment and could contribute to anti-tumor responses [28]. Evaluation of immune cell characterization and impact on tumor regression using the present study's treatment regimen is needed to further elucidate mechanisms contributing to enhanced tumor regression in the combination treatment group. Although no significant differences were shown in hypoxia or the vascular maturation index, previous studies using longitudinal imaging have indicated that trastuzumab can reduce hypoxia and increase vascular perfusion $[17,29]$. It is possible, by employing longitudinal imaging methods, windows of heightened oxygenation could be identified and that appropriately altered order of dosing could enhance treatment response in vivo.

Limitations of this study include the lack of longitudinal data evaluating microenvironmental changes corresponding with tumor growth dynamics. Longitudinal imaging methods such as Immuno-PET and dynamic contrast enhanced magnetic resonance imaging are alternative methods to quantitatively assess immune changes and vascularity of the microenvironment. However, neither of these methods would allow for histological validation at every time point. The present study used a single dose of radiation and provided preliminary data showing a lesser dose of radiation could be used in combination with trastuzumab to achieve the same therapeutic efficacy as a higher dose of radiation. Further studies 
should be conducted evaluating efficacy with a clinical treatment regimen employing fractionated dosing. Another possible limitation is due to the limited selection of trastuzumab dose. Additional trastuzumab treatment doses were investigated for the in vitro experiments in this study. Doses higher than $0.01 \mathrm{ng} / \mathrm{ml}$ exhibited effects on cell proliferation in single agent treatment groups; however, they did not affect combination treatment response. Doses lower than $0.01 \mathrm{ng} / \mathrm{ml}$ had no significant effect on HER2+ cell growth. Therefore, a sub-therapeutic dose of trastuzumab $(0.01 \mathrm{ng} / \mathrm{ml})$ was chosen as the appropriate treatment condition to assess combination effects in vitro. It is possible using a higher seeding density would allow for evaluation of cell proliferation with a higher trastuzumab dose, although when cells become confluent, the methods used to quantifying cell number become less accurate [21].

\section{Conclusions}

This study demonstrates longitudinal assessment of growth dynamics can result in different conclusions than end point assays and differences in methodologies should be taken into consideration when evaluating cellular response to treatment. Furthermore, this study yielded preliminary data that using trastuzumab in combination with radiation has the potential to decrease the dose of radiation administered without affecting therapeutic efficacy. Histology data revealed treatment with trastuzumab increased immune infiltration compared to radiation alone. Further assessment of immune changes in single agent and combination regimens could identify mechanistic explanations for differences in therapeutic efficacies.

\section{Abbreviations}

EGFP, enhanced green fluorescent protein; Gy, gray; HER2, human epidermal growth factor receptor 2; HRP, horseradish peroxidase; H\&E, hematoxylin and eosin; MTT, 3-[4,5-dimethylthiazolyl-2]-2,5diphenyltetrazolium bromide; SMA, smooth muscle actin; TBST, tris-buffered saline.

\section{Declarations}

\section{Ethics approval and consent to participate}

All procedures were approved by our institution's animal care and use committee.

\section{Consent for publication}

Not applicable.

\section{Availability of data and materials}

The data used in the current study as well as codes used for analyses are available from the corresponding author upon reasonable request.

\section{Competing interests}


The authors declare that they have no competing interests.

\section{Funding}

We thank the National Cancer Institute for support through U01CA174706 and R01CA186193. We thank the Cancer Prevention and Research Institute of Texas (CPRIT) for funding through RR160005. T.E.Y. is a CPRIT Scholar of Cancer Research. We thank funding from the Research Scholar Grant, RSG-18-006-01CCE from the American Cancer Society.

\section{Authors' contributions}

MJB, JV, AGS, and TEY designed the study. MJB and PNS performed experimentation. MJB analyzed the data and wrote the manuscript. All authors read and approved the final manuscript.

\section{Acknowledgements}

The authors would like to thank Dr. Amy Brock at the University of Texas at Austin for gifting the EGFPBT474 and EGFP-MDA-MB-231 cell lines used in this study.

\section{References}

1. Street W Breast Cancer Facts \& Figures 2017-2018. 44

2. Ménard S, Pupa SM, Campiglio M, Tagliabue E (2003) Biologic and therapeutic role of HER2 in cancer. Oncogene 22:6570-6578

3. Nahta R, Esteva FJ (2006) HER2 therapy: molecular mechanisms of trastuzumab resistance. Breast Cancer Res 8:215

4. Mignot F, Ajgal Z, Xu H, Geraud A, Chen JY, Mégnin-Chanet F, Kirova Y (2017) Concurrent administration of anti-HER2 therapy and radiotherapy: Systematic review. Radiotherapy and Oncology 124:190-199

5. Liang K, Lu Y, Jin W, Ang KK, Milas L, Fan Z (2003) Sensitization of breast cancer cells to radiation by trastuzumab. Mol Cancer Ther 2:1113-1120

6. Pietras RJ, Poen JC, Gallardo D, Wongvipat PN, Lee HJ, Slamon DJ (1999) Monoclonal antibody to HER-2/neureceptor modulates repair of radiation-induced DNA damage and enhances radiosensitivity of human breast cancer cells overexpressing this oncogene. Cancer research 59:1347-1355

7. Guo G, Wang T, Gao Q, Tamae D, Wong P, Chen T, Chen W-C, Shively JE, Wong JY, Li JJ (2004) Expression of ErbB2 enhances radiation-induced NF- $\mathrm{k}$ B activation. Oncogene 23:535-545

8. Hou J, Zhou Z, Chen X, et al (2016) HER2 reduces breast cancer radiosensitivity by activating focal adhesion kinase in vitro and in vivo. Oncotarget 7:45186-45198

9. Rao GS, Murray S, Ethier SP (2000) Radiosensitization of human breast cancer cells by a novel ErbB family receptor tyrosine kinase inhibitor. International Journal of Radiation 
Oncology*Biology*Physics 48:1519-1528

10. Gonzalez-Angulo AM, Litton JK, Broglio KR, et al (2009) High risk of recurrence for patients with breast cancer who have human epidermal growth factor receptor 2-positive, node-negative tumors 1 cm or smaller. J Clin Oncol 27:5700-5706

11. Spector NL, Blackwell KL (2009) Understanding the Mechanisms Behind Trastuzumab Therapy for Human Epidermal Growth Factor Receptor 2-Positive Breast Cancer. JCO 27:5838-5847

12. Vaupel P, Kelleher DK, Höckel M (2001) Oxygen status of malignant tumors: pathogenesis of hypoxia and significance for tumor therapy. Semin Oncol 28:29-35

13. Liu Z, Semenza GL, Zhang H (2015) Hypoxia-inducible factor 1 and breast cancer metastasis. J Zhejiang Univ Sci B 16:32-43

14. Lundgren K, Holm C, Landberg G (2007) Hypoxia and breast cancer: prognostic and therapeutic implications. Cell Mol Life Sci 64:3233-3247

15. Diehn M, Cho RW, Lobo NA, et al (2009) Association of Reactive Oxygen Species Levels and Radioresistance in Cancer Stem Cells. Nature 458:780-783

16. Godet I, Shin YJ, Ju JA, Ye IC, Wang G, Gilkes DM (2019) Fate-mapping post-hypoxic tumor cells reveals a ROS-resistant phenotype that promotes metastasis. Nat Commun 10:1-18

17. Sorace AG, Syed AK, Barnes SL, Quarles CC, Sanchez V, Kang H, Yankeelov TE (2017) Quantitative [18F]-FMISO- PET imaging shows reduction of hypoxia following trastuzumab in a murine model of HER2+ breast cancer. Mol Imaging Biol 19:130-137

18. Tsoutsou PG, Belkacemi Y, Gligorov J, Kuten A, Boussen H, Bese N, Koukourakis MI, area (AROME) on behalf of the A of R and $O$ in the M (2010) Optimal Sequence of Implied Modalities in the Adjuvant Setting of Breast Cancer Treatment: An Update on Issues To Consider. The Oncologist 15:1169-1178

19. Jakštys B, Ruzgys P, Tamošiūnas M, Šatkauskas S (2015) Different Cell Viability Assays Reveal Inconsistent Results After Bleomycin Electrotransfer In Vitro. J Membrane Biol 248:857-863

20. Menyhárt O, Harami-Papp H, Sukumar S, Schäfer R, Magnani L, de Barrios O, Győrffy B (2016) Guidelines for the selection of functional assays to evaluate the hallmarks of cancer. Biochimica et Biophysica Acta (BBA) - Reviews on Cancer 1866:300-319

21. Single A, Beetham H, Telford BJ, Guilford P, Chen A (2015) A Comparison of Real-Time and Endpoint Cell Viability Assays for Improved Synthetic Lethal Drug Validation. J Biomol Screen 20:1286-1293

22. Mátés L, Chuah MKL, Belay E, et al (2009) Molecular evolution of a novel hyperactive Sleeping Beauty transposase enables robust stable gene transfer in vertebrates. Nat Genet 41:753-761

23. Kowarz E, Löscher D, Marschalek R (2015) Optimized Sleeping Beauty transposons rapidly generate stable transgenic cell lines. Biotechnol J 10:647-653

24. Bliss $\mathrm{Cl}$ (1956) The calculation of microbial assays. Bacteriol Rev 20:243-258

25. Holleczek B, Stegmaier C, Radosa JC, Solomayer E-F, Brenner H (2019) Risk of loco-regional recurrence and distant metastases of patients with invasive breast cancer up to ten years after diagnosis - results from a registry-based study from Germany. BMC Cancer 19:520 
26. NCCN Guidelines for Patients $®$ | Invasive Breast Cancer. https://www.nccn.org/patients/guidelines/breast-invasive/46/index.html. Accessed 29 Oct 2019

27. Wang Z, Huang Y, Zhang J (2014) Molecularly targeting the PI3K-Akt-mTOR pathway can sensitize cancer cells to radiotherapy and chemotherapy. Cell Mol Biol Lett 19:233-242

28. Bloom, MJ, Jarrett, AM, Triplett, TA, Syed AK, Davis T, Yankeelov TE, Sorace AG (2020) Anti-HER2 induced myeloid cell alterations correspond with increasing vascular maturation in a murine model of HER2+ breast cancer. BMC Cancer 20(1):359

29. Sorace AG, Quarles CC, Whisenant JG, Hanker AB, Mclntyre JO, Sanchez VM, Yankeelov TE (2016) Trastuzumab improves tumor perfusion and vascular delivery of cytotoxic therapy in a murine model of HER2+ breast cancer: preliminary results. Breast Cancer Res Treat 155:273-284

\section{Figures}

a)

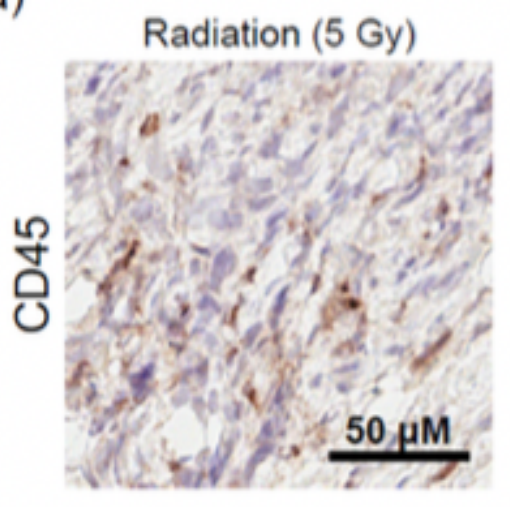

Trastuzumab

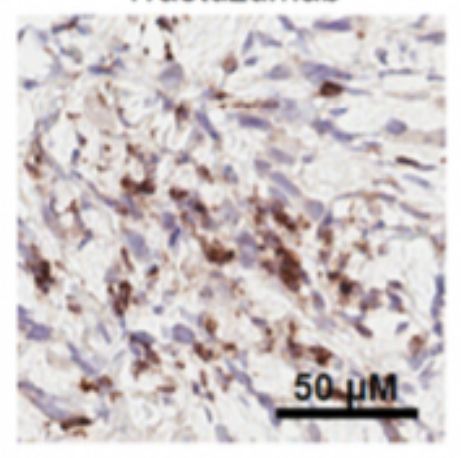

Trastuzumab + Radiation (5 Gy)

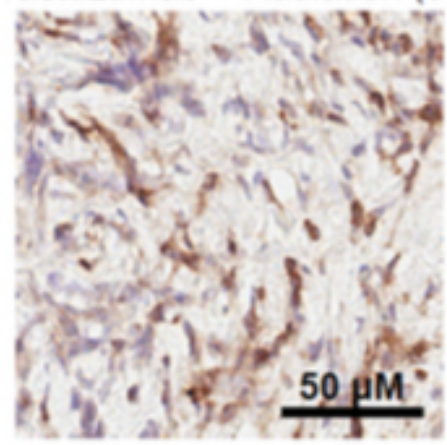

b)

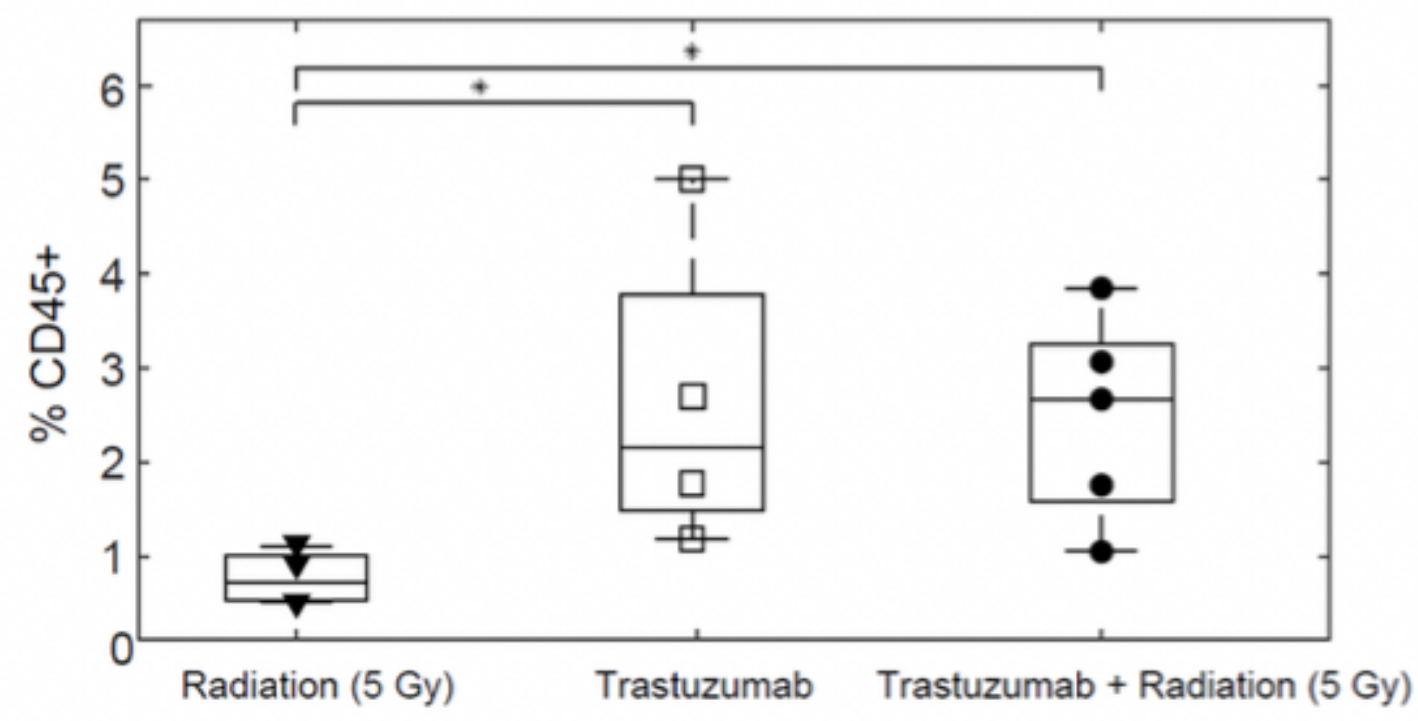




\section{Figure 1}

Percent CD45+ in single agent radiation, trastuzumab and combination treated tumors on Day 7. All radiation single agent and combination groups were treated with $5 \mathrm{~Gy}$ of radiation on Day 0 . All trastuzumab single agent and combination groups were treated with $10 \mathrm{mg} / \mathrm{kg}$ of trastuzumab on Days 0 and 3. a) Representative images of CD45 staining in single agent and combination treatment groups. b) Percent CD 45+ staining from central slice of tumors in single agent and combination treatment groups, revealing significant increases in CD45+ in trastuzumab single agent and trastuzumab + radiation (5 Gy) compared to radiation alone $(P=0.03)$. No significant difference was seen in mice treated with single agent trastuzumab and trastuzumab + radiation $(P=1.00)$.

a)

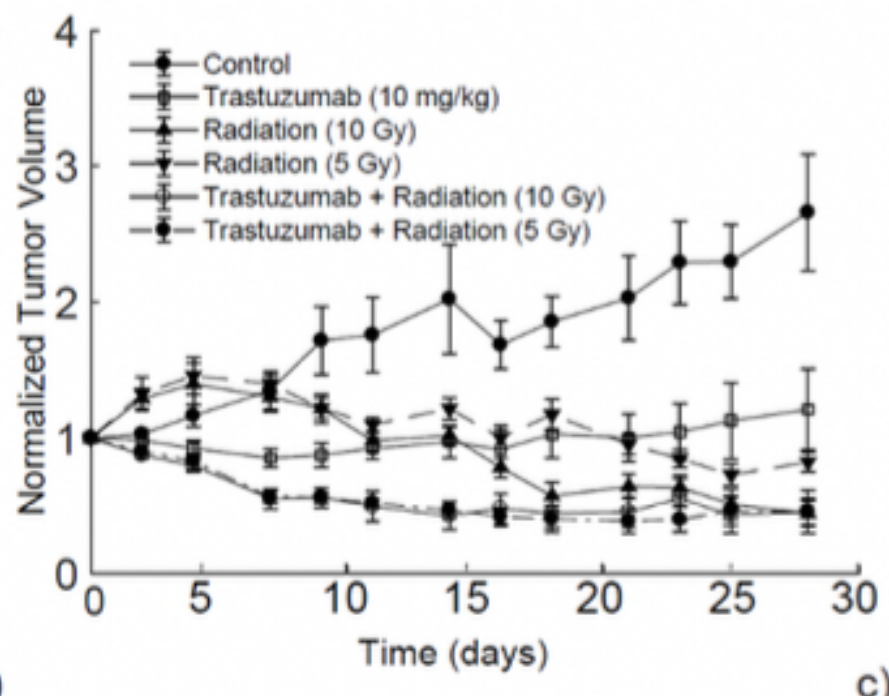

b)

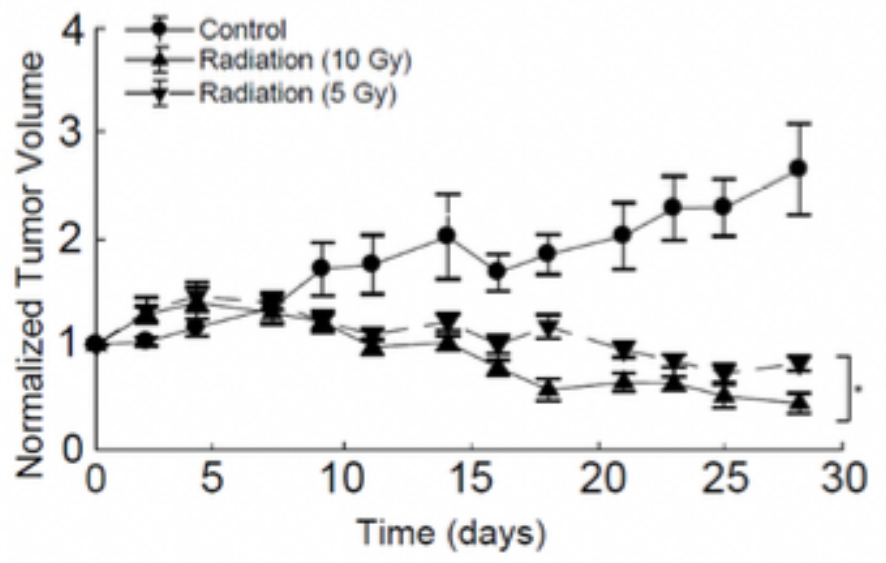

c)

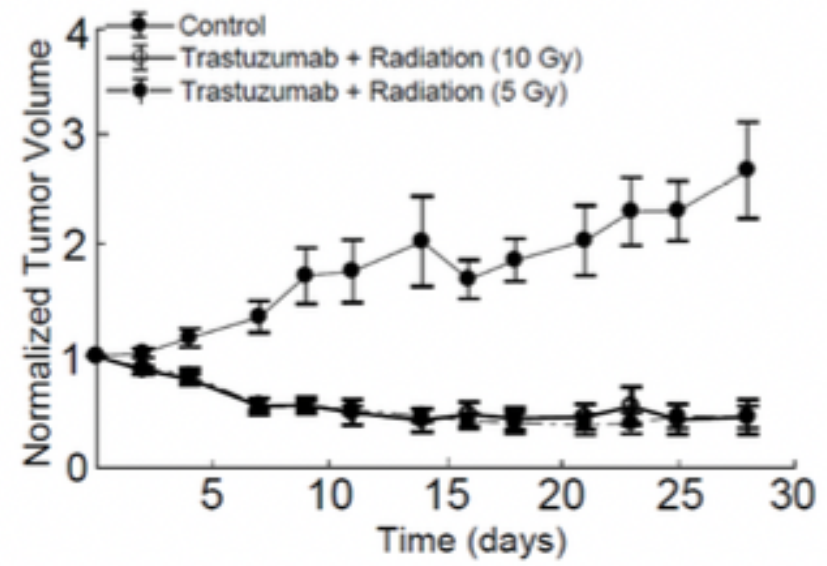

Figure 2

In vivo tumor growth response to single agent and combination trastuzumab and radiation therapy. All mice were treated with radiation on Day 0 and trastuzumab on Days 0 and 3. a) Displays all groups. b) Displays control with single agent radiation therapy. There was a significant difference between groups treated with single agent $5 \mathrm{~Gy}$ and single agent $10 \mathrm{~Gy}$ radiation $(\mathrm{P}<0.001)$. c) Displays control with 
trastuzumab + radiation (5 Gy) and trastuzumab + radiation (10 Gy). There was no significant difference in radiation treated groups when adding trastuzumab to the regimen $(P=0.56)$.

a)

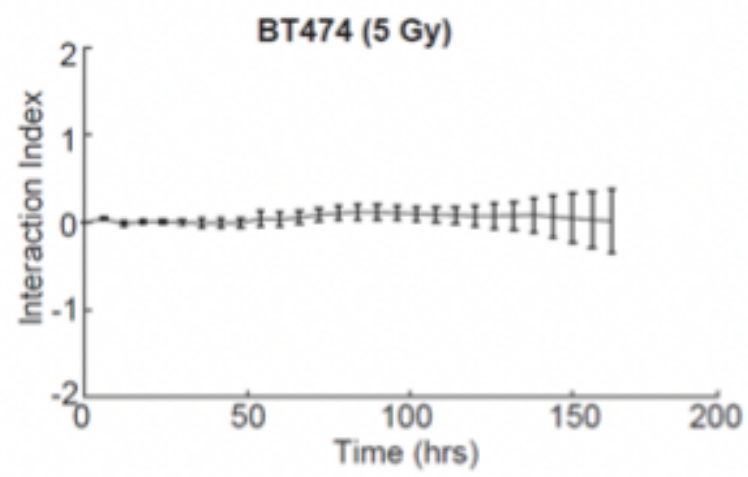

c)

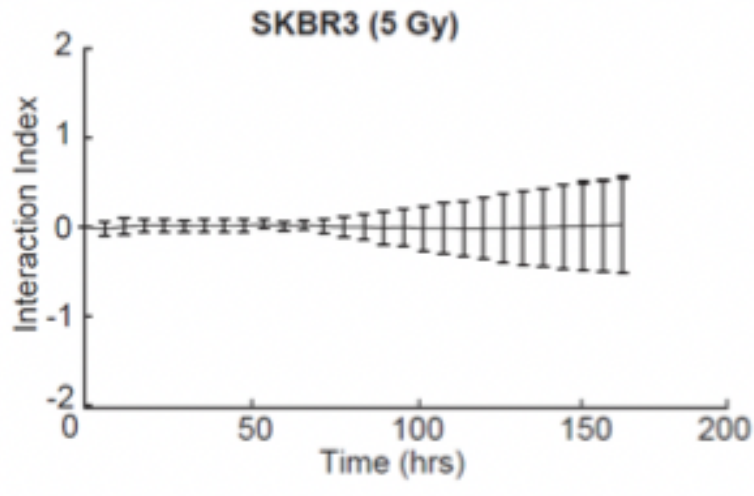

e)

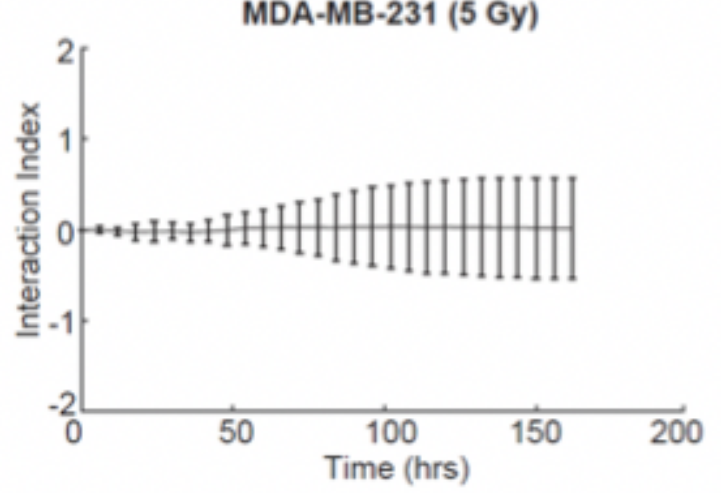

b)

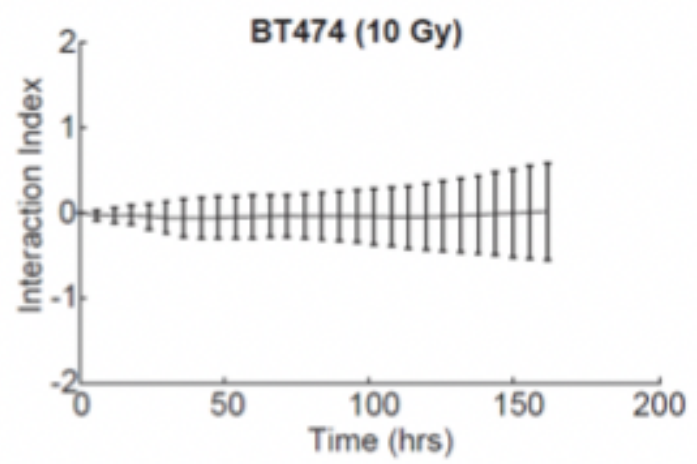

d)

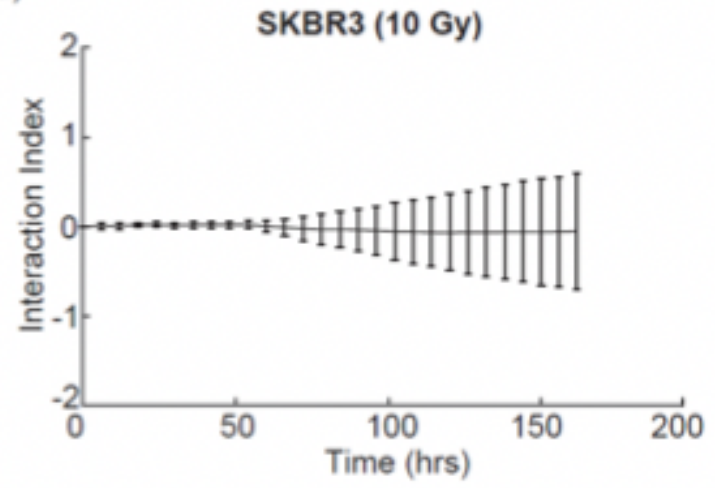

f)

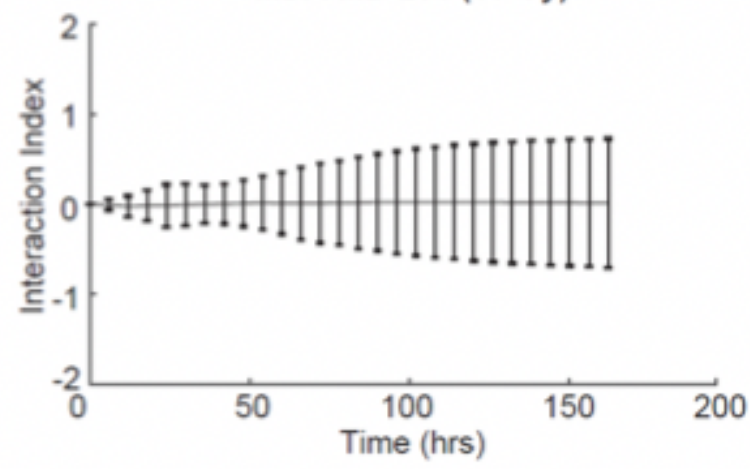

\section{Figure 3}

Interaction index calculated per time point over one week of treatment. All groups were treated with 0.01 $\mathrm{ng} / \mathrm{ml}$ of trastuzumab. Graphs display interaction index of a) BT474 cells, 5 Gy radiation + trastuzumab, b) BT474 cells, 10 Gy radiation + trastuzumab, c) SKBR3 cells, 5 Gy radiation + trastuzumab, d) SKBR3 cells, 10 Gy radiation + trastuzumab, e) MDA-MB-231 cells, 5 Gy radiation + trastuzumab and f) MDA-MB231 cells, 10 Gy radiation + trastuzumab. No group had an interaction index significantly above or below 0 at any time point, indicating additive treatment affects. 
a)

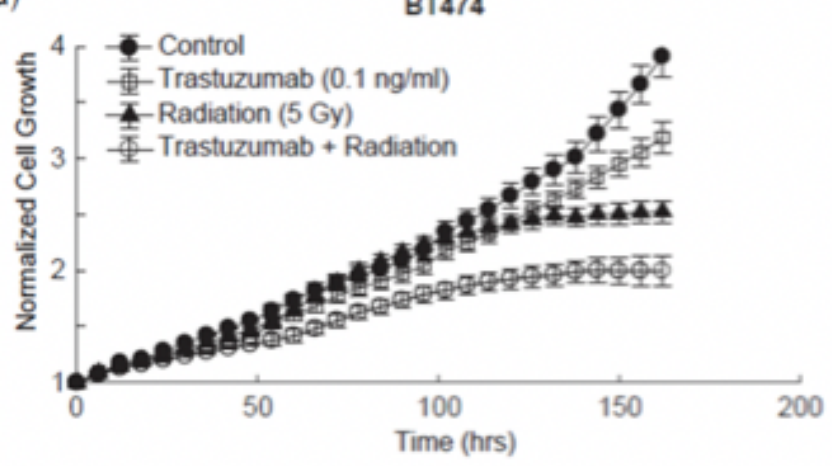

c)

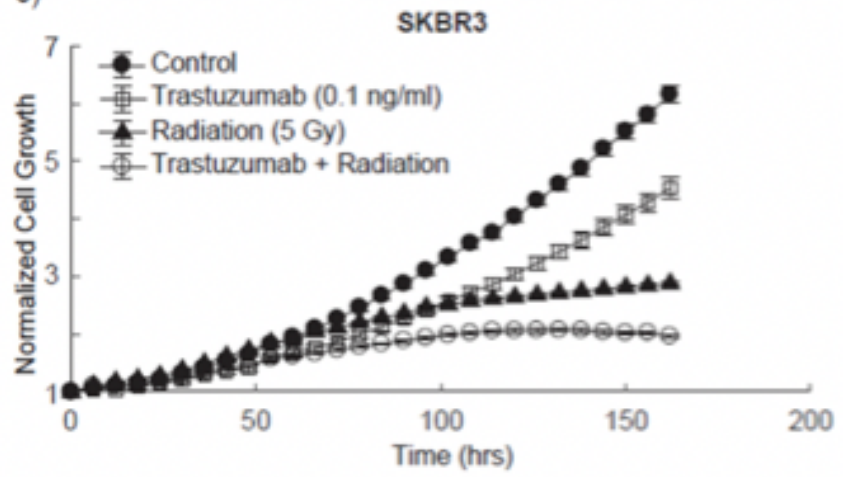

e)

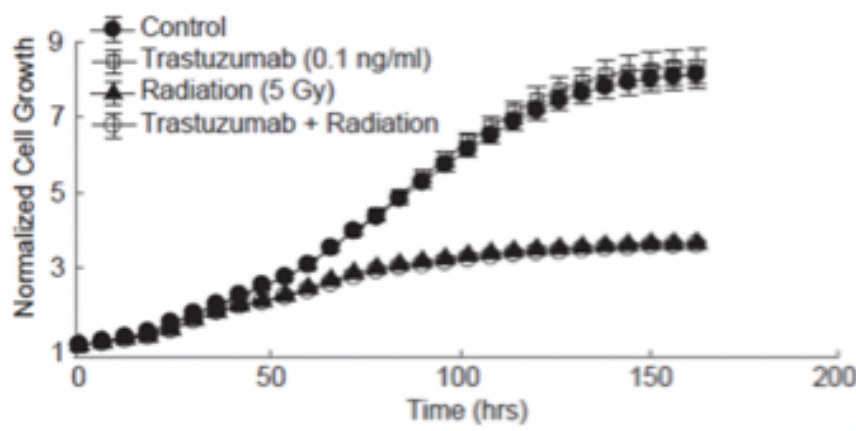

f) b)

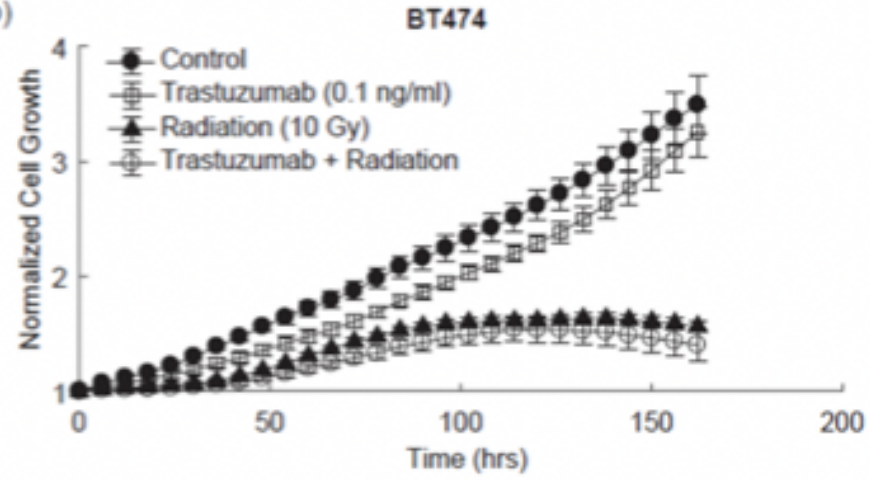

d)
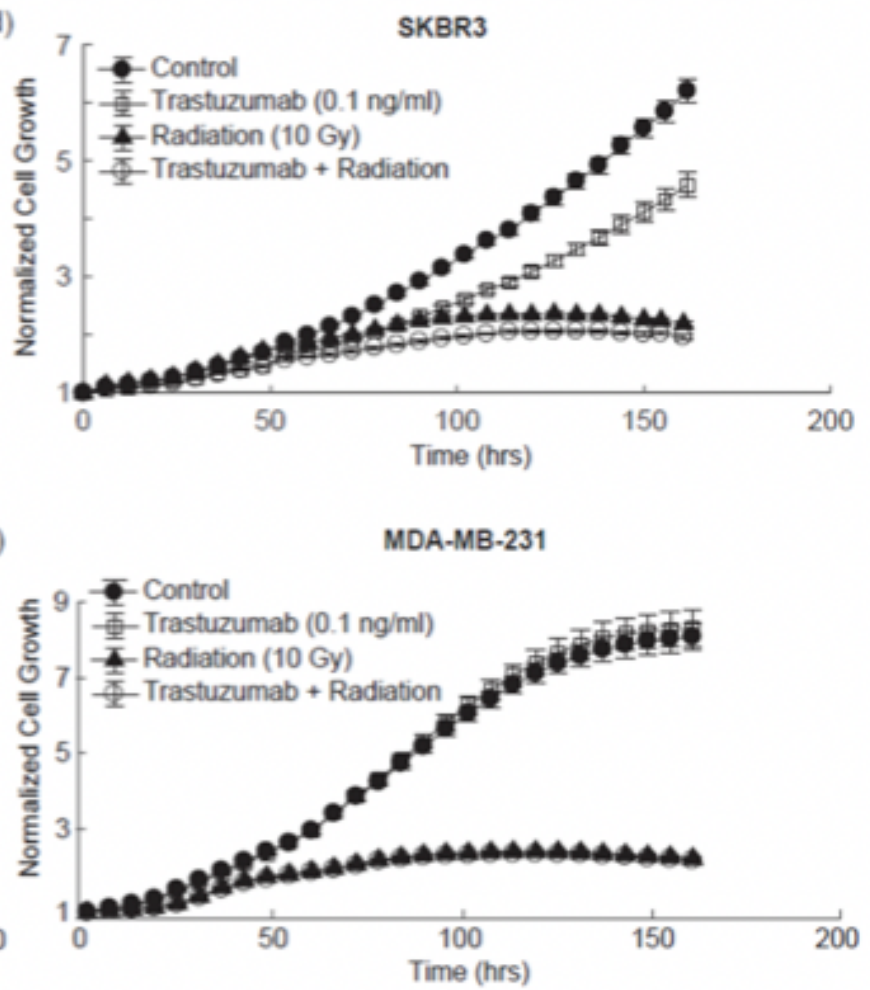

Figure 4

Cell proliferation over one week after radiation, trastuzumab or combination treatment. All trastuzumab single agent and combination groups were treated with $0.1 \mathrm{ng} / \mathrm{ml}$ of trastuzumab from 0-48 hours. All radiation single agent and combination groups were treated with 5 or 10 Gy radiation on Day 0 . Graphs display proliferation of: a) BT474 cells after trastuzumab, 5 Gy radiation and combination treatment, b) BT474 cells after trastuzumab, 10 Gy radiation and combination treatment, c) SKBR3 cells after trastuzumab, 5 Gy radiation and combination treatment, d) SKBR3 cells after trastuzumab, 10 Gy radiation and combination treatment, e) MDA-MB-231 cells after trastuzumab, 5 Gy radiation and combination treatment, f) MDA-MB-231 cells after trastuzumab, 10 Gy radiation and combination treatment. No significant difference was observed in control MDA-MB-231 cell proliferation and cells treated with single agent trastuzumab $(P=0.88$, e \& $f)$. No significant difference in cell proliferation was 
observed between cells treated with single agent radiation and cells treated with combination treatment $(P=0.84$, e \& $P=0.80, f)$.

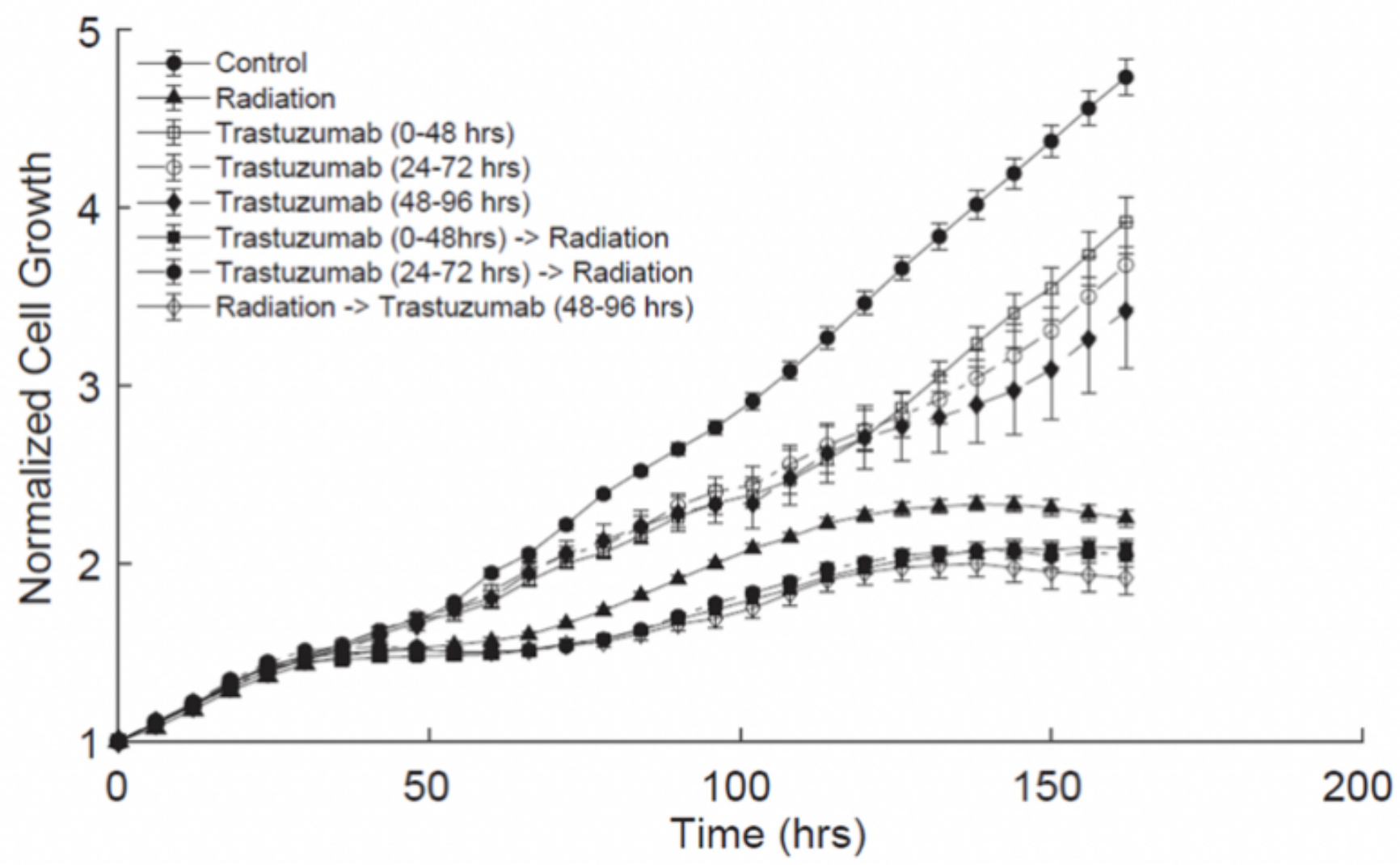

Figure 5

Differences in BT474 cell growth with trastuzumab given before, simultaneously, and after radiation therapy. Treatment with trastuzumab before radiation did not significantly alter cell response to therapy compared to treatment of trastuzumab at the same time $(P=1.00)$ or treatment after radiation $(P=0.98)$. Simultaneous treatment with trastuzumab did not significantly alter cell response to therapy compared to treatment after radiation $(P=0.93)$. Altering the order of dosing of trastuzumab and radiation did not alter cell response to treatment in vitro. 
a) Experiment 1: Determine if order of radiation and trastuzumab therapy alters cell response

1. Trastuzumab BEFORE Radiation

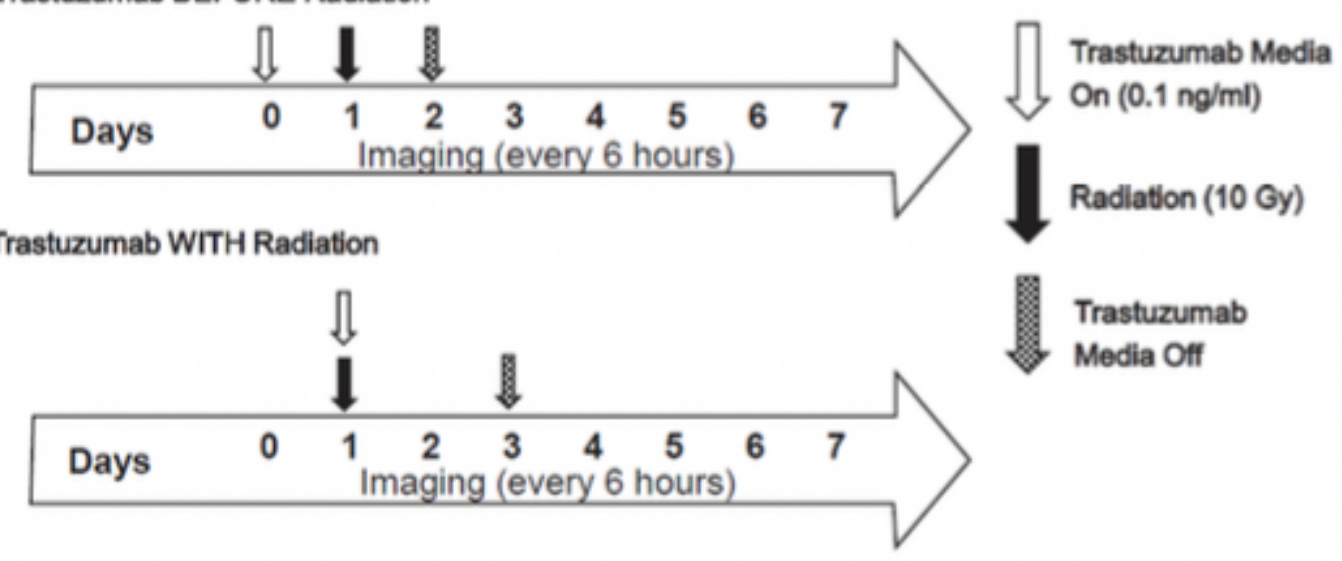

3. Trastuzumab AFTER Radiation

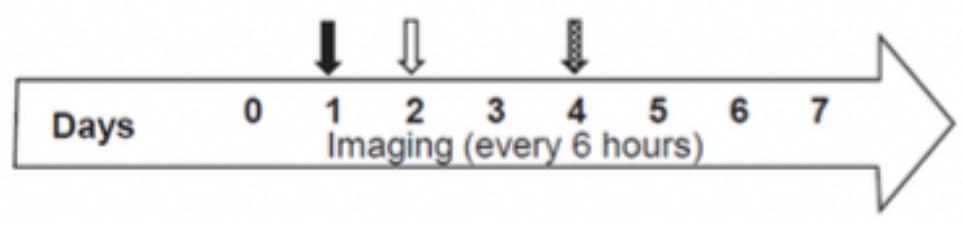

b) Experiment 2: Quantify radiation + trastuzumab combination effects

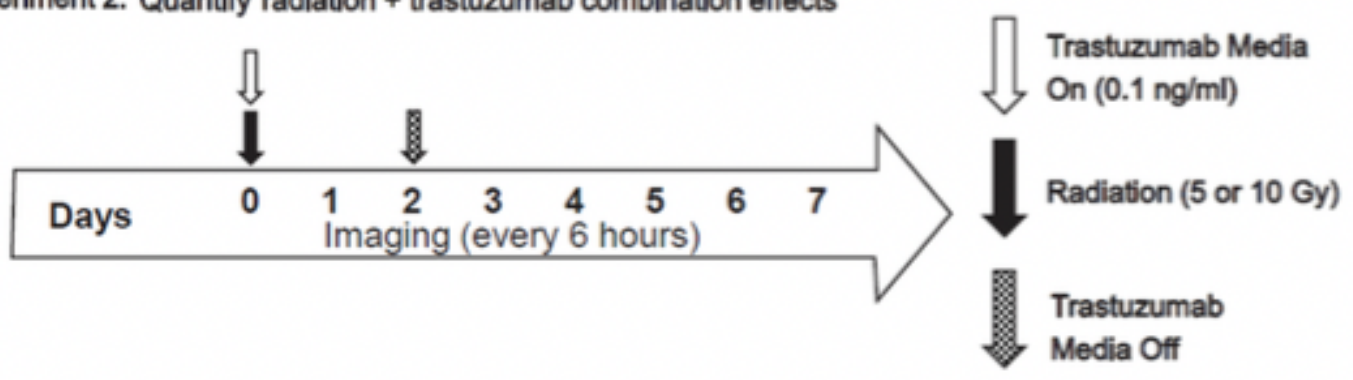

\section{Figure 6}

Treatment plans for in vitro experiments. a) Cells were treated with either trastuzumab $(0.1 \mathrm{ng} / \mathrm{ml})$ from 048 hours ( 1 , before radiation), 24-72 hours ( 2 , with radiation) or 48-96 hours ( 3 , after radiation). All groups received radiation (10 Gy) at 24 hours. All groups were imaged every 6 hours for seven days. b) Cells were treated with trastuzumab $(0.1 \mathrm{ng} / \mathrm{ml})$ and radiation ( 5 or $10 \mathrm{~Gy}$ ) at the start of the experiment (Day 0 ). Trastuzumab was removed 48 hours later. Cells were imaged every six hours for seven days.

\section{Supplementary Files}

This is a list of supplementary files associated with this preprint. Click to download.

- SuppFigures.docx 